\title{
Intellectualized Identifying and Precision Control System for Horticultural Crop Diseases Based on Small Unmanned Aerial Vehicle*
}

\author{
Hongxin Cao ${ }^{1, * *}$, Yuwang Yang ${ }^{2}$, Zhiyuan $\mathrm{Pei}^{3}$, Wenyu Zhang ${ }^{1}$, Daokuo $\mathrm{Ge}^{1}$, \\ Yiran Sha ${ }^{1,4}$, Weixin $\mathrm{Zhang}^{1}$, Kunya Fu ${ }^{1}$, Yan Liu ${ }^{1}$, Yuli Chen ${ }^{1}$, \\ Hongjun Dai ${ }^{1}$, and Hainan Zhang $^{2}$ \\ ${ }^{1}$ Institute of Agricultural Economics and Information Research/Engineering Research Center \\ for Digital Agriculture, Jiangsu Academy of Agricultural Sciences, \\ Nanjing 210014, Jiangsu, P.R. China \\ caohongxin@hotmail.com \\ ${ }^{2}$ School of Computer Sciences, Nanjing University of Sciences and Technology, \\ Nanjing 210014, Jiangsu Province, P.R. China \\ ${ }^{3}$ Resource Monitoring Station, Chinese Academy of Agricultural Engineering, \\ Beijing 100026, P.R. China \\ ${ }^{4}$ College of Applied Meteorology, Nanjing University of Information Science \\ and Technology, Nanjing 210044, Jiangsu Province, P.R. China \\ caohongxin@hotmail.com
}

\begin{abstract}
To explore small unmanned aerial vehicle (UAV) remote sensing identifying technology for horticultural crop diseases, and to combine it with small UAV spraying, the peach leaf blade was taken as material, the peach shot-hole disease was taken as object for spectral capture, and the intellectualized identifying and precision control system for horticultural crop diseases were developed, mainly including the identifying system for horticultural crop diseases, spraying pesticide system carried by small UAV, and the ground mobile control station system. Some sub-systems were initially tested in laboratory and outside. This study can provide an important reference for unmanned precise control of horticultural crop diseases.
\end{abstract}

Keywords: Spectrum, identification, horticultural crop diseases, precision control, unmanned aerial vehicle (UAV).

\section{$1 \quad$ Introduction}

The control for diseases and insect pests is the key to horticultural crop production with high yielding, good quality, better benefit, ecology, and safety, and the traditional

\footnotetext{
* Foundation Information: Jiangsu Province Agricultural Scientific Technology Innovation Fund [CX(11)2042], and Jiangsu Province Scientific Technology Supporting Program [BE2012386-1].

** Corresponding author.
}

D. Li and Y. Chen (Eds.): CCTA 2012, Part II, IFIP AICT 393, pp. 196-202, 2013.

(C) IFIP International Federation for Information Processing 2013 
control for diseases and insect pests depends on manned or machined application of pesticide, which has some obvious disadvantages such as bigger work intensity, high cost, and environmental problems, etc. [1-4].

In recent years, rapid progress on the studies of unmanned aerial vehicle (UAV) spraying facility has been made in Japan and USA, etc. The first UAV in the world was created by Yamaha Company in Japan in 1990, which was mainly used in bestrewing pesticide [5]. Since 90's in 20 century, the remote control helicopter has been used in prevention and cure for diseases and insect pests of field crops, fruit tree, and vegetables, proper fertilization and quality management of field crops, and estimation of crop growth status (rice) [6, 7]. Because the UAV has some advantages such as small in scale, agility in grasp, and good effect in spray, etc., it has been into one of development trend that the UAV is used in agricultural production. The studies of aviation spraying of UAV were also done in USA [8]. In china, the application of UAV spraying is not adaptable with needs of modern agricultural development, and has a big gap comparing with that of overseas [8].

The objectives of this study is to explore the UAV remote sensing identifying technology for horticultural crop diseases, to combine it with UAV spraying, and to create intellectualized identifying and precision control system for horticultural crop diseases based on small UAV.

\section{Materials and Methods}

\subsection{Materials}

The experiment for spectral capture was carried out in peach garden of headquarters in Jiangsu Academy of Agricultural Sciences from May to Nov. in 2012, the peach leaf blade was taken as material, and the peach shot-hole disease was taken as object for spectral capture.

\subsection{Methods}

Canopy spectral measurement: The spectral reflectance of the disease leaf and the happy leaf in the same peach, and the disease peach and the happy peach in the same peach garden and time were measured using MSR16R Multispectral Radiometry (16 wave bands, spectral ranges from $460 \mathrm{~nm}$ to $1700 \mathrm{~nm}$, and manufactured by Cropscan, Inc. in USA) in the fine day's 10:00 a.m.-2:00 p.m. with above $45^{\circ}$ solar elevation angle, the radiometry probe surface was paralleled to peach canopy or target leaf with $0.2 \mathrm{~m}$ upright distance, and the spectral reflectance adjusting of standard whiteboard was done around each measurement.

Expert identification of disease leaf: The severity of disease is composed of the size and number of disease spot in a leaf or a peach, in that the size and number were divided into 3 levels according to expert identification.

Analysis and using of data: First, the ranges of sensitive waveband for disease parameters were found based on correlation between disease parameters and single 
waveband spectral reflectance of disease leaf and peach, and the vegetation indices (VI) composed of sensitive waveband were computed based on existing research; Second, the optimum VI of the most sensitivity to disease parameters were screened out through analyzing correlation between disease parameters and VI, and the corresponding regression equations were established, in that correlation analysis, and VI computing were done using OFFICE.2007 and SPSS v 16.0.

\subsection{Concept Design}

First, the standard spectral reflectance of disease leaf in peach garden was captured using spectral radiometry, and the spectral reflectance of peach garden canopy was measured using spectral probe carried by small UAV. Second, the information captured was wirelessly sent to ground, analysed, and identified. Third, the intellectualized identifying and precision control system for horticultural crop diseases based on identifying-pesticide spraying integration was set up through combining the spectrum-based identifying for crop diseases and the UAV-based pesticide spraying system (Fig. 1).

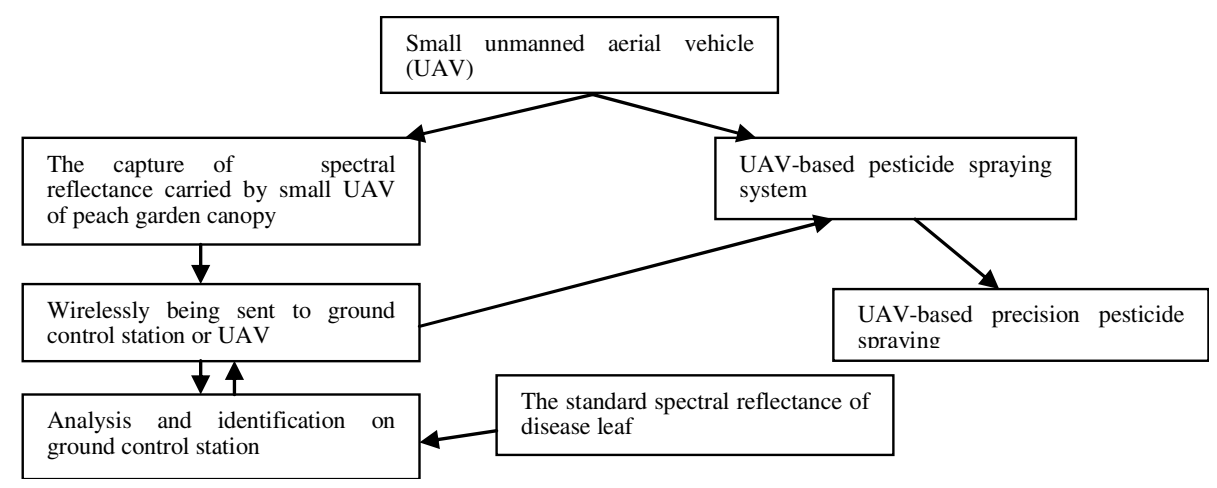

Fig. 1. Concept design of the intellectualized identifying and precision control system for horticultural crop diseases based on identifying- pesticide spraying integration

\section{Results}

The intellectualized identifying and precision control system for horticultural crop diseases based on small UAV mainly included identifying system for horticultural crop diseases, spraying pesticide system carried by small UAV, and the ground mobile control station system.

\subsection{Identifying System for Horticultural Crop Diseases}

The identifying system included the part carried by small UAV, and the ground mobile control station. 


\section{The Spectral Measurement Device Carried by Small UAV for Horticultural Crop} Diseases. The focal plane probe with $200 \mathrm{~mm}$ large caliber, inclined $45^{\circ}$ lens structure, large light area, and stable turning was used in spectral probe, and the head of spectral measurement probe was composed of the imaging optics system, the bearing flat, and spectral probe. The scanning spectral ranges from visible to infra-red (430-1030 nm, 60 wavebands with $10 \mathrm{~nm}$ resolution) were selected, its verification examples were shown in Fig.2. The linear detector-scanning program was applied in modularizing spectral meter system, and the spectral resolution, space resolution, sensitivity, and volume and weight were considered in the development of spectral measurement section. The GPS system was applied in UAV positioning, and the dynamic precision of position system can go to the meter level. The peg-top stable flat was introduced into UAV in order to insure pose stability of the spectral measurement device.
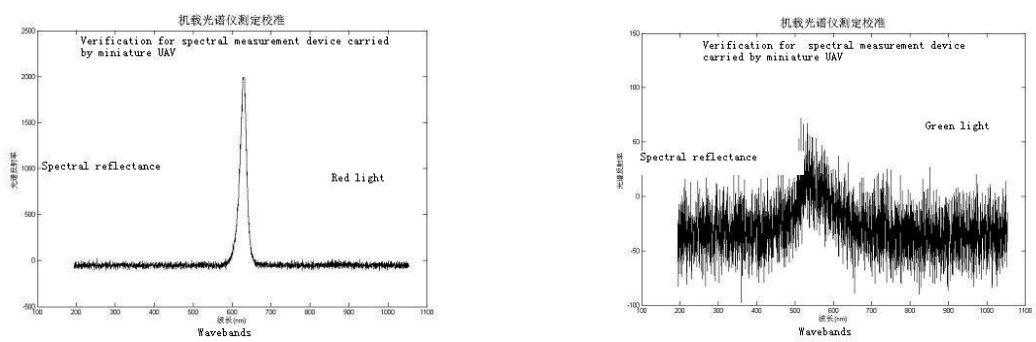

Fig. 2. Verification for the spectral measurement device carried by small UAV

\section{Communication between the Small UAV and the Ground Mobile Control Station.}

Due to the large number of spectral data needed to be wirelessly transferred between the small UAV and the ground mobile control station, the bandwidth for communication should be larger. At the same time, the various statements from the ground mobile control station needed to be incepted by UAV, but because the formats of statements were to a certainty, its length was finitude, and the demand to communication bandwidth was not high. The independent uplink and downlink channel were adopted by the system, and the asymmetric bandwidths were used in transfer to avoid interfere between data and statements transfer each other. Under the normal condition, this can provide less than 3.5 Mbps of the uplink speed, and $54 \mathrm{Mbps}$ of the downlink speed. The communication frequency with $2.4 \mathrm{GHz}$ was selected, and the wireless transceiver system developed by ourselves was used in communication device.

The Real-Time Transaction for Spectral Data and Identifying of Peach Diseases. There is a powerful computer system in the ground mobile control station, which mainly was used in pre-processing for the spectral data, such as GPS data processing and composite, calibration processing, bad line repair, pixel registration, histogram calculation, contrast stretching, regional amplification, spectral curve display, data capture, and data format conversion, etc. The spectral data from the UAV was real-timely processed, the comparison of the final spectral data and the standard 
spectral reflectance of disease leaf were done after a series of processing steps, and the identifying information of peach diseases were obtained.

\subsection{Spraying Pesticide System Carried by Small UAV}

The impact factors of UAV spraying effect included spraying device, target plant, atomization degree for droplet, droplet flow transport properties, and droplet penetrability, etc.

The Development of UAV Spraying Device. It included self-regulation for amount of spray, droplet diameter, and nozzle structure, etc. so as to fulfill low volume, ultra-low volume, and electrostatic spraying, and to help to realize double times atomization when the high speed airflow were passed through spraying nozzle.

Droplet Atomization. It can be divided into two modes, centrifugal type and hydraulic atomization. The centrifugal atomization nozzle was selected in UAV spraying device, and driven by generator in UAV. The size of droplet can be regulated using rotate speed and the dial structure of nozzle.

In addition, the atomization droplet was effected by airflow speed, state, and outside atmosphere, etc. in the flight UAV. The droplet atomization was studied in order to obtain the optimum particle size and movement state.

Sedimentation Process. In the droplet transport and sedimentation processes, due to space of flow was very complex, and it let droplets to collide and polymerize each other, which resulted in movement of droplets with huge randomicity. Therefore, the mathematic models of space of flow were developed through experiments in order to capture the integrative flow state of droplets in the space of flow.

Effects of Meteorological Conditions on Spraying. The optimum time of spraying was one of important factors for raising droplet target rate, and under the different regions, and terrain conditions, the effects of temperature and wind speed, swirl from rub of airflow and crop, etc. on distribution of droplet on crop were studied.

Effects of Droplet Size and Number on Spraying. The droplet coverage was related to many factors, especially rotor vortex, and flight velocity in UAV. The droplet size depends on the target state sprayed, the optimization of droplet size can help to raise target coverage, and use pesticide availably.

Establishment of UAV Operating Instructions for Spraying Technology. The UAV operating instructions for spraying technology were established according to the relevant FAO's rule to ensure security for flight and operators, spraying device stability, and operating precision, and reduce the negative effects on environment, nearby crop, and human and livestock.

\subsection{The Ground Mobile Control Station System}

The ground mobile control station system included the ground mobile control center carried by vehicle, the ground remote control and measurement system for UAV, the 
ground control station system for spraying pesticide, and the ground spectral signal incept system, etc., in that the ground remote control and measurement system for UAV were composed of industrial control computer, remote control transmitter, telemetry receiver, remoter, and power box, etc.

\subsection{Test of the System}

Test of the system mainly included the identifying precision of peach diseases, and the spraying pesticide effect, and the spraying pesticide effect was checked through comparison the spraying pesticide position carried by UAV and the observed disease position in peach garden. Some sub-systems were initially tested in laboratory and outside.

\section{Discussion and Conclusions}

\subsection{Discussion}

Identifying System for Horticultural Crop Diseases. The previous studies showed that there were some shape and physiological changes in leaf such as becoming yellow, wilt, and withered, etc. when plant were stressed by diseases, which was visible changes easily to be identified. In addition, red light, infra-red, "blue shift", and "red shift", etc. all need to be considered in this study [9].

Size and Volume of Device Carried by Small UAV. In order to reduce loading of the small UAV, the spectral data captured by small UAV were transferred to the ground control station, and real-timely processed in the ground control station. The optic structure of spectral system carried by the UAV were elaborateelaborately designed, the whole spectral system weight was less than $1 \mathrm{~kg}$, and its volume was less than 0.001 $\mathrm{m}^{3}(0.10 \mathrm{~m} \times 0.10 \mathrm{~m} \times 0.10 \mathrm{~m})$.

Spraying Technology in Lower Level. The spraying technology in lower level is the key to the intellectualized identifying and precision control system for horticultural crop diseases based on small UAV. Therefore, the centrifugal atomization nozzle was used in small UAV spraying device to adapt to spraying pesticide in lower level, and raise control efficiency.

The System Need to be Test Under Different Conditions. The study is still in the initial stage, the various sub-systems were not integrated into the intellectualized identifying and precision control system for horticultural crop diseases, and tested under different conditions.

\subsection{Conclusions}

The intellectualized identifying and precision control system for horticultural crop diseases were developed, mainly including the identifying system for horticultural crop 
diseases, spraying pesticide system carried by small UAV, and the ground mobile control station system. Some sub-systems, e.g. the spectral measurement device carried by small UAV, were initially tested in laboratory and outside.

\section{References}

1. Zhang, L.: The Pollution of Agricultural Environment with Chemical Pesticide and its Control. J. Nanjing Agric. Technol. Coll. 17(4), 36-38. 43 (2001)

2. State Environmental Protection Administration: China's Pesticide Pollution and Its Being Problems and Suggestions. Environ. Prot. 6, 23-24 (2001)

3. Zhu, M.Y., Zhang, J.X., Lu, Z.H., et al.: Controlling the Environmental Pollution of Chemical Pesticide, Carrying out Consecutions Protection. Jiangxi Plant Prot. 26(3), 29-31 (2003)

4. Tu, Y.Q.: Thinking of Problems of Agriculture Chemicals and Circumstance. Reclaiming and Rice Cultiv. 1, 58-60 (2003)

5. Zhang, Y.: Application of remote control helicopter in Japan agriculture. World Agric. (4), 49-50 (1997)

6. Sun, X.Y.: Multi-purposes unmanned helicopter in agriculture. Shangdong Agric. Mech. (9), 27 (1999)

7. Hunt, E.R.: Evaluation of digital photography from model aircraft for remote sensing of crop biomass and nitrogen status. Precis. Agric. 6, 359-378 (2005)

8. Gong, Y., Fu, X.M.: Aviation spraying pesticide technology in modern agriculture. Agric. Equip. Technol. 34(6), 26-29 (2008)

9. Huang, M.Y., Wang, J.H., Huang, W.J., et al.: Hyperspectral character of stripe rust on winter wheat and monitoring by remote sensing. Trans. Chin. Soc. Agric. Eng. 19(6), 154-155 (2003) 